\title{
Robust Watermarking Scheme Using Wave Atoms
}

\author{
H. Y. Leung and L. M. Cheng \\ Department of Electronic Engineering, City University of Hong Kong, Kowloon, Hong Kong \\ Correspondence should be addressed to H. Y. Leung, leunghonyin@gmail.com
}

Received 8 July 2010; Accepted 17 September 2010

Academic Editor: Dennis Deng

Copyright ( $\odot 2011 \mathrm{H}$. Y. Leung and L. M. Cheng. This is an open access article distributed under the Creative Commons Attribution License, which permits unrestricted use, distribution, and reproduction in any medium, provided the original work is properly cited.

\begin{abstract}
A robust blind watermarking scheme using wave atoms is proposed. The watermark is embedded in the wave atom transform domain by modifying one of the scale bands. The detection and extraction procedures do not need the original host image. We tested the proposed algorithm against common image processing attacks like JPEG compression, Gaussian noise addition, median filtering, and salt and pepper noise, and also compared its performance with other watermarking schemes using multiscale transformation. They were carried out using Matlab software. The experimental results demonstrate that the proposed algorithm has great robustness against various imaging attacks.
\end{abstract}

\section{Introduction}

Since the rapid development of digital technology and internet, it makes anyone possible to create, replicate, transmit, and distribute digital content in an effortless way [1]. Thus, how to protect the copyright of these digital protections efficiently has been a hot issue in the recent two decades. As a copyright protection technology, digital watermarking recently draws a lot of attention since it can embed desirable information in transmitted audio, image, and video data files and also ensures the data integrity at the same time [2].

A digital watermark should have two main properties, which are robustness and imperceptibility. Robustness means that the watermarked data can withstand different image processing attacks and imperceptibility means that the watermark should not introduce any perceptible artifacts [1]. According to whether the original image is needed or not during the detection, watermarking methods can be sorted as nonblind, semiblind, or blind [3]. Nonblind technique requires the original image; semiblind technique only requires the watermark; blind technique requires neither the original image nor the watermark.

In the past two decades, discrete wavelet transformation, discrete Fourier transformation (DFT), and discrete cosine transformation (DCT) are mainly used in digital watermarking due to the robustness requirement [4-6]. In 2006, Demanet [7] introduced a new multi-scale transform called wave atoms. It can be used to effectively represent warped oscillatory functions [8]. Oriented textures have a significantly sparser expansion in wave atoms than in other fixed standard representations like Gabor filters, wavelets, and curvelets. Many existing applications of wave atom transform show its great potential for image denoising $[9,10]$. However, there are few researches on finding out the feasibility of wave atom transform applying in digital watermarking. It would be interesting to investigate whether wave atom transform is suitable for watermarking.

Sensitivity of human eye to noise in textured area is less and it is more near the edges according to the HVS characteristics [11]. Therefore, little modifications of textures area are usually imperceptible by human eyes, and the wave atom can provide significantly sparser expansion for the oscillatory functions or oriented textures [8]. Thus, modifying significant wave atom coefficients may result in little image quality degradation.

In this paper, we present a blind watermarking method using the wave atom transform. And the robustness tests for the proposed method and comparisons with other watermarking schemes are also described. This paper is organized as follows. In Section 2, wave atom transform is presented. 
The details of embedding and extracting approaches are given in Section 3. The experimental results are described in Section 4. Finally, Section 5 provides the conclusion.

\section{Wave Atom Transform}

Demanet [7] introduced wave atoms, that can be seen as a variant of $2 \mathrm{D}$ wavelet packets and obey the parabolic scaling law, that is, wavelength $\sim\left(\right.$ diameter $^{2}$. They prove that oscillatory functions or oriented textures (e.g., fingerprint, seismic profile, and engineering surfaces) have a significantly sparser expansion in wave atoms than in other fixed standard representations like Gabor filters, wavelets, and curvelets.

Wave atoms have the ability to adapt to arbitrary local directions of a pattern and to sparsely represent anisotropic patterns aligned with the axes. The elements of a frame of wave packets $\left\{\phi_{u}(x)\right\}, x \in \mathbb{R}^{2}$, are called wave atoms (WAs) when there is a constant $C_{M}$ such that

$$
\begin{aligned}
\left|\hat{\phi}_{u}\right| \leq & C_{M} 2^{-j}\left(1+2^{-j}\left|\omega-\omega_{u}\right|\right)^{-M} \\
& +C_{M} 2^{-j}\left(1+2^{-j}\left|\omega+\omega_{u}\right|\right)^{-M}
\end{aligned}
$$

and $\left|\phi_{u}\right| \leq C_{M} 2^{j}\left(1+2^{j}\left|x-x_{u}\right|\right)^{-M}$, with $M=1,2, \ldots$ The hat denotes Fourier transformation and the subscript $u=$ $\left(j, m_{1}, m_{2}, n_{1}, n_{2}\right)$ of integer-valued quantities index a point $\left(x_{u}, \omega_{u}\right)$ in phase space as

$$
\begin{gathered}
x_{u}=\left(x_{1}, x_{2}\right)_{\mu}=2^{-j}\left(n_{1}, n_{2}\right), \\
\omega_{u}=\left(\omega_{1}, \omega_{2}\right)_{\mu}=\pi 2^{j}\left(m_{1}, m_{2}\right),
\end{gathered}
$$

where $C_{A} 2^{j} \leq \max _{k=1,2}\left|m_{k}\right| \leq C_{B} 2^{j}$, with $C_{A}$ and $C_{B}$ positive constants whose values depend on the numerical implementation. Hence, the position vector $x_{\mu}$ is the center of $\phi_{u}(x)$, and the wave vector $\omega_{u}$ denotes the centers of both bumps of $\hat{\phi}_{u}(\omega)$.

The parabolic scaling is encoded in the localization conditions as follows [12]: at scale $2^{-2 j}$, the essential frequency support is of size $\sim 2^{-j}$. The subscript $j$ denotes different dyadic coronae and the subscripts $\left(m_{1}, m_{2}\right)$ label the different wave number $\omega_{u}$ within each dyadic corona.

In fact, WAs are constructed from tensor products of 1D wavelet packets. The family of real-valued $1 \mathrm{D}$ wave packets is described by $\psi_{m_{1}, n_{1}}^{j}\left(x_{1}\right)$ functions, where $j \geq 0, m_{1} \geq 0$, and $\psi_{m_{1}, n_{1}}^{j}\left(x_{1}\right)=2^{j / 2} \psi_{m_{1}}^{0}\left(2^{j} x_{1}-n_{1}\right)$ with

$$
\begin{aligned}
\hat{\psi}_{m_{1}}^{0}\left(\omega_{1}\right)=e^{-i \omega / 2}\left\{e^{-i \alpha_{m 1}} g\left[\epsilon_{m 1}\left(\omega_{1}-\pi m_{1}-\frac{\pi}{2}\right)\right]\right. \\
\left.+e^{-i \alpha_{m 1}} g\left[\epsilon_{m 1+1}\left(\omega_{1}+\pi m_{1}+\frac{\pi}{2}\right)\right]\right\},
\end{aligned}
$$

where $\epsilon_{m 1}=(-1)^{m_{1}}$ and $\alpha_{m 1}=\left(2 m_{1}+1\right) \pi / 4$. The function $g$ is an appropriate real-valued $C^{\infty}$ bump function, compactly supported on an interval of length $2 \pi$ and chosen such that

$$
\sum_{m}\left|\hat{\psi}_{m_{1}}^{0}(\xi)\right|^{2}=1 .
$$

The $2 \mathrm{D}$ extension is formed by the products

$$
\begin{gathered}
\phi_{u}^{+}\left(x_{1}, x_{2}\right)=\psi_{m_{1}}^{j}\left(x_{1}-2^{-j} n_{1}\right) \psi_{m_{2}}^{j}\left(x_{2}-2^{-j} n_{2}\right), \\
\phi_{u}^{-}\left(x_{1}, x_{2}\right)=H \psi_{m_{1}}^{j}\left(x_{1}-2^{-j} n_{1}\right) H \psi_{m_{2}}^{j}\left(x_{2}-2^{-j} n_{2}\right),
\end{gathered}
$$

where $H$ is the Hilbert transform and $\mu=\left(j, m_{1}, m_{2}, n_{1}, n_{2}\right)$. The recombinations $\phi_{u}^{(1)}=\left(\phi_{u}^{+}+\phi_{u}^{-}\right) / 2$ and $\phi_{u}^{(2)}=\left(\phi_{u}^{+}-\right.$ $\left.\phi_{u}^{-}\right) / 2$ form the WA frame. A numerical implementation of WAs using the Matlab software is provided in [13].

\section{Proposed Method}

Suppose that $I$ and $w$ denote the host image of size $M \times N$ and binary watermark of size $n \times n$, respectively. The host image is decomposed into four subimages as follows:

$$
\begin{array}{cl}
I_{1}(i, j)=I(i, j), & I_{2}(i, j)=I\left(i, \frac{N}{2}+j\right), \\
I_{3}(i, j)=I\left(\frac{M}{2}+i, j\right), & I_{4}(i, j)=I\left(\frac{M}{2}+i, \frac{N}{2}+j\right),
\end{array}
$$

where $i=1,2, \ldots, M / 2, j=1,2, \ldots, N / 2$, and $I_{1}, I_{2}, I_{3}$, and $I_{4}$ denote the four subimages.

3.1. The Embedding Procedure. The proposed watermark embedding scheme is shown in Figure 1. Our proposed method is based on the idea of paper [14] proposed by Zhu and Sang. Their method modifies the DC components of discrete cosine transform (DCT) domain using quantification to embed watermark; however the quantification approach is rather complicated and less effective, and all DC coefficient values are utilized. In our case, we propose to use wave-atom coefficients with a much more simplified quantization approach with only two levels for each bit embedded, and only selective coefficients are used for modification purpose giving better susceptibility against attacks. The embedding process is described as follows.

(1) Divide the original image $I$ of size $M \times N$ to form four subimages, $I_{1}, I_{2}, I_{3}$, and $I_{4}$, using (6).

(2) Wave-atom Transform is then applied to the four subimages. Accordingly, these subimages are decomposed into five bands in our case. The fourth-scale band is selected to embed watermark $w$.

(3) Select the coefficients $C_{u}$ from the sets $S_{1}, S_{2}, S_{3}$, and $S_{4}$ whose absolute values are smaller than $r$ to modify and label as $D_{u}$, where $u=\left(j, m_{1}, m_{2}, n_{1}, n_{2}\right)$ of integer-valued quantities index is a point $\left(x_{u}, \omega_{u}\right)$ in phase space.

(4) Suppose that $Z_{u}=D_{u} \bmod Q$. The function mod computes modulus after division. $Q$ is a quantification threshold for adjusting watermark embedding 


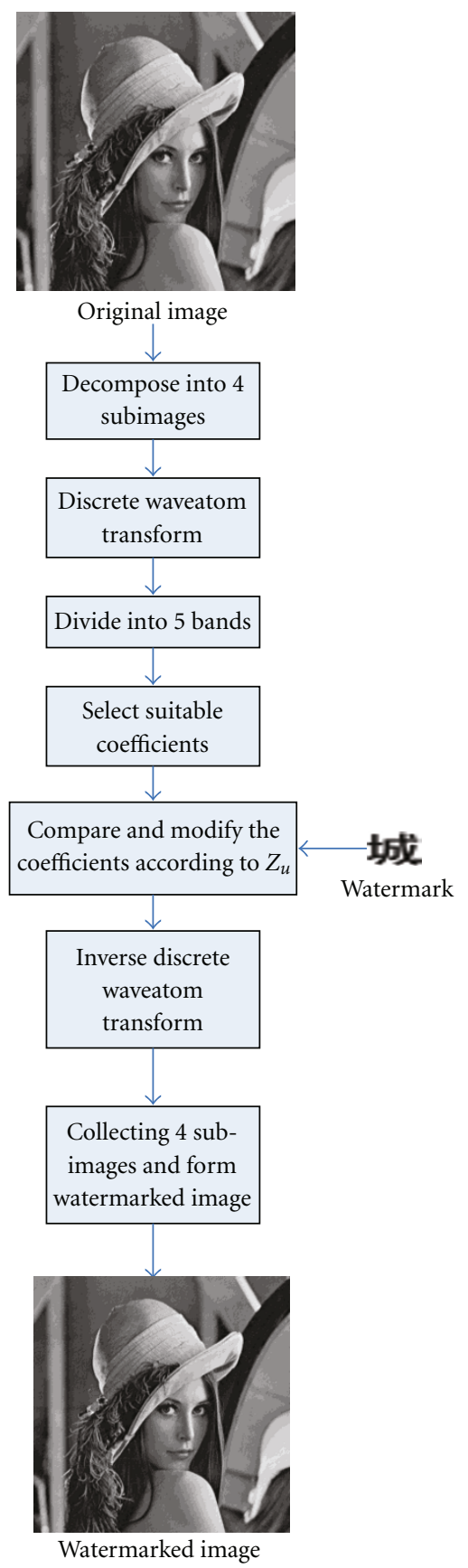

FIgURE 1: The embedding procedure.

depth and can affect the watermarked image quality and the robustness of the embedded watermark. If $Q$ is too small, embedding watermark robustness will be worse; if $Q$ is too large, it will degrade the quality of the watermarked image, and, therefore, $Q$ is chosen properly based on the detailed application condition of watermark. In our proposed method, one wave atom wedge is used for embedding one bit. Thus, more than one coefficient will get modified in the wedge and they represent the same bit. Assume that the length of watermark bits is $l$.

When embedding bit $w_{c}=0$,

$$
D_{u}= \begin{cases}D_{u}+\frac{Q}{4}-Z_{u} & \text { if } Z_{u} \in\left[0, \frac{3 Q}{4}\right), \\ D_{u}+\frac{5 Q}{4}-Z_{u} & \text { if } Z_{u} \in\left[\frac{3 Q}{4}, Q\right) .\end{cases}
$$

When embedding bit $w_{c}=1$,

$$
D_{u}= \begin{cases}D_{u}-\frac{Q}{4}-Z_{u} & \text { if } Z_{u} \in\left[0, \frac{Q}{4}\right), \\ D_{u}+\frac{3 Q}{4}-Z_{u} & \text { if } Z_{u} \in\left[\frac{Q}{4}, Q\right),\end{cases}
$$

where $c=1,2, \ldots, l$.

(5) Repeat the above process until embedding all bits and apply the inverse wave-atom transform to the modified coefficients sets.

(6) Obtain the output watermarked image $I^{\prime}$ by collecting 4 modified subimages.

3.2. The Extracting Procedure. Suppose that $I^{\prime}$ is the watermarked image for watermark detection. When extracting the watermark sequence, our watermarking model does not need the original image. The proposed watermark extraction scheme is shown in Figure 2. The extracting process is described as follows.

(1) Divide $I^{\prime}$ to four subimages, $I_{1}^{\prime}, I_{2}^{\prime}, I_{3}^{\prime}$, and $I_{4}^{\prime}$, using (6).

(2) Wave-atom transform is then applied to subimages $I_{1}^{\prime}, I_{2}^{\prime}, I_{3}^{\prime}$, and $I_{4}^{\prime}$ to obtain four coefficients sets, $S_{1}^{\prime}$, $S_{2}^{\prime}, S_{3}^{\prime}$, and $S_{4}^{\prime}$.

(3) Similar to the embedding phase, watermark is extracted from the fourth scale band. First, select coefficient $C_{u}^{\prime}$ within the sets $S_{1}^{\prime}, S_{2}^{\prime}, S_{3}^{\prime}$, and $S_{4}^{\prime}$ whose absolute values are smaller than $r$ to modify and label as $D_{u}^{\prime}$, where $u=\left(j, m_{1}, m_{2}, n_{1}, n_{2}\right)$ of integer-valued quantities index is a point $\left(x_{u}, \omega_{u}\right)$ in phase space.

(4) Calculate $Z_{u}^{\prime}=D_{u}^{\prime} \bmod Q$. Let $h$ denote the number of coefficient $D_{u}^{\prime}$ inside a wave atom wedge $\delta_{j, m 1, m 2}$. The watermark sequence $t_{c}$ is extracted as follows. For a nonempty wedge $\delta_{j, m 1, m 2}$,

$$
t_{c}(k)= \begin{cases}0 & \text { if } Z_{u}^{\prime} \in\left[0, \frac{Q}{2}\right), \\ 1 & \text { if } Z_{u}^{\prime} \in\left[\frac{Q}{2}, Q\right),\end{cases}
$$

where $k=1,2, \ldots, h$ and $c=1,2, \ldots, l$.

A sequence $t_{c}$ is obtained, which is used for extracting correct watermark bits.

(5) Finally, the watermark $w_{c}$ can be reconstructed as follows:

$w_{c}= \begin{cases}0 & \text { if number of bit } 0 \text { in } t_{c}>\text { number of bit } 1 \text { in } t_{c}, \\ 1 & \text { if number of bit } 1 \text { in } t_{c} \geq \text { number of bit } 0 \text { in } t_{c},\end{cases}$

where $c=1,2, \ldots, l$. 


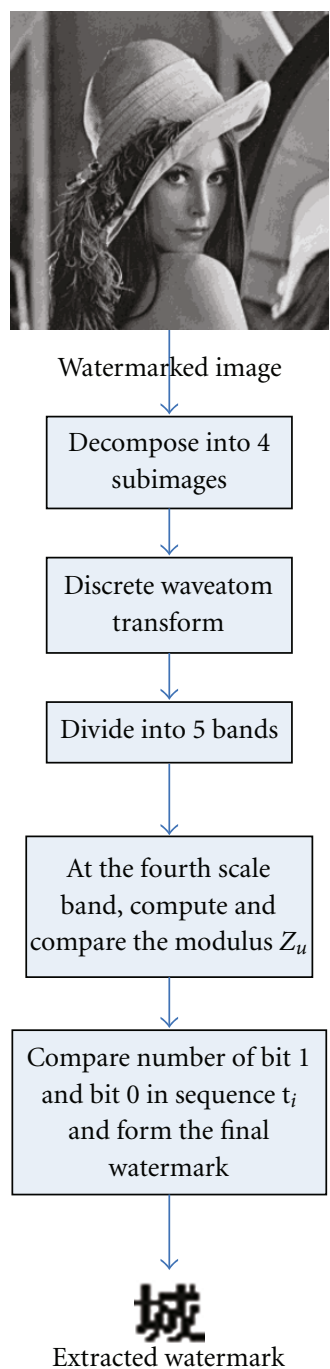

FIGURE 2: The extracting procedure.

TABLE 1: The values of PSNR.

PSNR value of watermarked lena image $(\mathrm{dB})$

\begin{tabular}{lc}
\hline Zhu and Sang [14] & 54.329 \\
Xiao et al. [16] & 44.5323 \\
Leung et al. [17] & 42.8072 \\
Tao and Eskicioglu [18] & 35.8 \\
Ni et al. [19] & 44.7 \\
Proposed scheme & 40.379
\end{tabular}

The proposed method is similar to the quantization index modulation- (QIM-) based watermarking schemes. QIM was first proposed by Chen and Wornell [15]. In Chen's method, there are two uniform quantizers $Q_{0}(s)$ and $Q_{1}(s)$ for watermark embedding, while we simplify the approach and use only one quantizer $Q$ which enhances the computation efficiency. Our step size of the proposed method is $Q / 2$. To embed the watermark, we shift the modulus values of waveatom coefficients to the median of the interval or to the nearest median of the neighbor intervals according to the watermark bit. If the values are within the desired interval, they need to be moved to the median of the same interval. However, if the values are placed in the undesired interval, they need to be shifted to the median of the nearest neighbor interval. Thus, the proposed simplified quantization index modulation approach can speed up the entire extraction process.

\section{Experimental Results}

The experimental results of the proposed watermarking scheme are presented in this section. In order to test the robustness of the proposed watermarking scheme, we used the $512 \times 512$ gray-scale image, Lena, shown in Figure 3(a) as the test image. The watermarked image is illustrated in Figure 3(b), which has good visual quality. The binary watermark is shown in Figure 3(c), whose size is $16 \times 16$. The extracted watermark is shown in Figure 3(d) with $\mathrm{NC}$ value $=1$ which shows the correct watermark extraction. Our experimental system is composed of an Intel Core-Quad CPU with a $2.66 \mathrm{GHz}$ core and $3 \mathrm{~GB}$ DDR2.

In the experiments, the quantification threshold $Q$ is 24 and the threshold of coefficient selection $r$ is 60 . The mean squared error (MSE) between the original and watermarked images is defined by

$$
\mathrm{MSE}=\frac{1}{M \cdot N} \sum_{i=1}^{M} \sum_{j=1}^{N}\left(I(i, j)-I^{\prime}(i, j)\right)^{2},
$$

where $I(i, j)$ and $I^{\prime}(i, j)$ denote the pixel value at position $(i, j)$ of the original image $I$ and the watermarked image $I^{\prime}$ with size of $M \times N$ pixels, respectively.

Hence, the watermarked image quality is represented by the peak signal-to-noise ratio (PSNR) between $I$ and $I^{\prime}$ and is calculated by

$$
\mathrm{PSNR}=10 \log _{10}\left(\frac{255^{2}}{\mathrm{MSE}}\right) \quad(\mathrm{dB}) .
$$

To evaluate the robustness of the algorithm, the normalized cross-correlation (NC) is employed. More similar watermarks will get a larger NC value. The $\mathrm{NC}$ between the embedded watermark, $W(i, j)$, and the extracted watermark $W^{\prime}(i, j)$ is defined by

$$
\mathrm{NC}=\frac{\sum_{i=1}^{M_{W}} \sum_{j=1}^{N_{W}}\left[W(i, j) \cdot W^{\prime}(i, j)\right]}{\sum_{i=1}^{M_{W}} \sum_{j=1}^{N_{W}}[W(i, j)]^{2}},
$$

where $M_{W}$ and $N_{W}$ denote the width and height of the watermark, respectively.

4.1. Robustness Tests. Several common signal processing attacks are applied to verify the robustness of the proposed scheme including Gaussian low-pass filtering, Gaussian additive noise, Laplacian image enhancement, JPEG compression, and salt and pepper noises. Furthermore, we compare the performance of the proposed scheme with other 
TABLE 2: Experiment results comparison under Gaussian noises (NC values).

\begin{tabular}{lccccccccccc}
\hline Standard variance of Gaussian noises & 6 & 8 & 10 & 12 & 14 & 16 & 18 & 20 & 25 & 30 \\
\hline Zhu and Sang [14] & 0.9254 & 0.8718 & 0.7912 & 0.7033 & 0.639 & 0.5844 & 0.5927 & 0.582 & 0.4947 & 0.4869 \\
Xiao et al. [16] & 0.9926 & 0.9778 & 0.9738 & 0.9778 & 0.955 & 0.963 & 0.9511 & 0.9403 & 0.9129 & 0.893 \\
Leung et al. [17] & 1 & 0.9926 & 0.9889 & 0.9853 & 0.9891 & 0.9553 & 0.9312 & 0.9315 & 0.8508 & 0.8596 \\
Tao and Eskicioglu [18] & 0.8584 & 0.822 & 0.7974 & 0.7772 & 0.7634 & 0.7538 & 0.7476 & 0.7405 & 0.731 & 0.7231 \\
Proposed scheme & 1 & 0.9816 & 0.9591 & 0.8226 & 0.6674 & 0.5333 & 0.5414 & 0.4926 & 0.4963 & 0.5319 \\
\hline
\end{tabular}

TABLE 3: Experiment results comparison under salt and pepper noises (NC values).

\begin{tabular}{lccccccccccc}
\hline Density parameter of "salt and pepper noises" & 0.02 & 0.04 & 0.06 & 0.08 & 0.1 & 0.12 & 0.14 & 0.16 & 0.18 & 0.2 \\
\hline Zhu and Sang [14] & 0.5986 & 0.4983 & 0.4776 & 0.5346 & 0.5291 & 0.5441 & 0.5235 & 0.4772 & 0.4433 & 0.4851 \\
Xiao et al. [16] & 0.9587 & 0.9024 & 0.8263 & 0.8196 & 0.7981 & 0.7856 & 0.7729 & 0.7407 & 0.7766 & 0.696 \\
Leung et al. [17] & 0.9093 & 0.8677 & 0.7916 & 0.7658 & 0.7648 & 0.6594 & 0.6971 & 0.6807 & 0.7213 & 0.6393 \\
Tao and Eskicioglu [18] & 0.9784 & 0.9579 & 0.9386 & 0.9209 & 0.9035 & 0.8869 & 0.8714 & 0.8559 & 0.8437 & 0.8301 \\
Proposed scheme & 0.5804 & 0.4605 & 0.5481 & 0.5284 & 0.5037 & 0.5299 & 0.5271 & 0.5821 & 0.5401 & 0.5004 \\
\hline
\end{tabular}

TABLE 4: Experiment results comparison under Laplacian sharpening (NC values).

\begin{tabular}{lccccccccccc}
\hline Laplacian parameter & 0.1 & 0.2 & 0.3 & 0.4 & 0.5 & 0.6 & 0.7 & 0.8 & 0.9 & 1 \\
\hline Zhu and Sang [14] & 0.7565 & 0.7565 & 0.7638 & 0.7721 & 0.7693 & 0.7783 & 0.7783 & 0.7884 & 0.7783 & 0.7794 \\
Xiao et al. [16] & 0.9963 & 0.9963 & 0.9963 & 0.9963 & 0.9963 & 0.9963 & 0.9963 & 0.9963 & 0.9963 & 0.9963 \\
Leung et al. [17] & 0.9963 & 0.9963 & 0.9963 & 0.9963 & 0.9963 & 0.9963 & 0.9963 & 0.9963 & 0.9963 & 0.9963 \\
Tao and Eskicioglu [18] & 0.7967 & 0.7975 & 0.8007 & 0.8028 & 0.8044 & 0.8083 & 0.8082 & 0.8095 & 0.8109 & 0.8215 \\
Proposed scheme & 0.6268 & 0.6256 & 0.6421 & 0.674 & 0.6643 & 0.6692 & 0.7015 & 0.6963 & 0.728 & 0.7253 \\
\hline
\end{tabular}

TABLe 5: Experiment results comparison under Jpeg compression (NC values).

\begin{tabular}{lcccccccccc}
\hline Jpeg compression parameter & 80 & 60 & 40 & 35 & 30 & 25 & 20 & 15 & 10 \\
\hline Zhu and Sang [14] & 1 & 1 & 0.9785 & 0.9813 & 0.7303 & 0.914 & 0.6407 & 0.7026 & 0.3899 & 0.7057 \\
Xiao et al. [16] & 0.9553 & 0.9093 & 0.9481 & 0.8657 & 0.8074 & 0.8955 & 0.7427 & 0.7454 & 0.6915 & 0.6519 \\
Leung et al. [17] & 1 & 1 & 0.9963 & 0.9963 & 1 & 0.9853 & 0.9704 & 0.9289 & 0.7761 & 0.6138 \\
Tao and Eskicioglu [18] & 0.9704 & 0.9245 & 0.891 & 0.881 & 0.8682 & 0.8558 & 0.8382 & 0.818 & 0.7858 & 0.7413 \\
Ni et al. [19] & 0.9547 & 0.7550 & 0.5314 & N/A & N/A & N/A & N/A & N/A & N/A & N/A \\
Proposed scheme & 0.9963 & 0.9813 & 0.9524 & 0.9403 & 0.9231 & 0.8889 & 0.8493 & 0.7427 & 0.6256 & 0.5735 \\
\hline
\end{tabular}

TABLE 6: Experiment results comparison under low-pass filtering (NC values).

\begin{tabular}{|c|c|c|c|c|c|}
\hline Standard variance (window) of "low-pass filtering" & $0.5(3)$ & $1.5(3)$ & $0.5(5)$ & $1.5(5)$ & $3(5)$ \\
\hline Zhu and Sang $[14]$ & 0.9214 & 0.8206 & 0.9214 & 0.9179 & 0.8422 \\
\hline Xiao et al. [16] & 0.9889 & 0.9706 & 0.9889 & 0.9299 & 0.8541 \\
\hline Leung et al. [17] & 1 & 1 & 1 & 1 & 1 \\
\hline Tao and Eskicioglu [18] & 0.9697 & 0.912 & 0.9695 & 0.8741 & 0.8582 \\
\hline Proposed scheme & 1 & 0.9926 & 0.9963 & 0.853 & 0.6114 \\
\hline
\end{tabular}

TABLE 7: Experiment results comparison under cropping (NC values).

\begin{tabular}{lccccc}
\hline Cropping & Type 1 (Figure 4(f)) & Type 2 (Figure 4(g)) & Type 3 (Figure 4(h)) & Type 4 (Figure 4(i)) & Type 5 (Figure 4(j)) \\
\hline Zhu and Sang [14] & 0.7262 & 1 & 0.7262 & 1 & 1 \\
Xiao et al. [16] & 0.8001 & 0.8756 & 0.8046 & 0.8095 & 0.853 \\
Leung et al. [17] & 0.9118 & 0.9158 & 0.8888 & 0.869 & 0.8748 \\
Tao and Eskicioglu [18] & 0.678 & 0.8611 & 0.6788 & 0.6758 & 0.6768 \\
Proposed scheme & 0.9889 & 0.9702 & 0.8074 & 0.8474 & 0.9058 \\
\hline
\end{tabular}




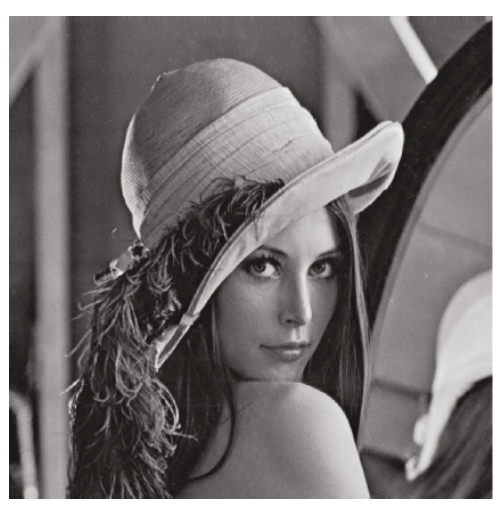

(a) Lena image

城

(c) Binary watermark

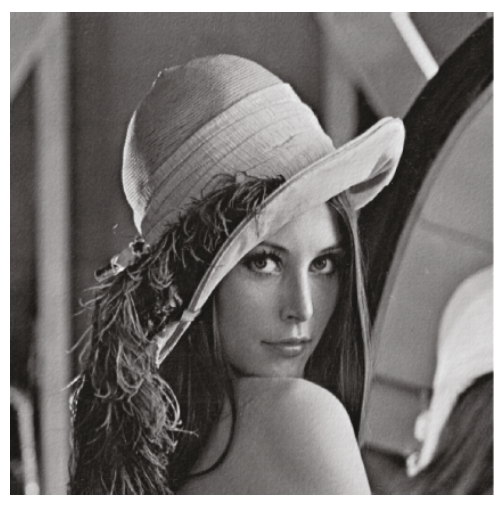

(b) Watermarked Lena image

城

(d) Extracted watermark with $\mathrm{NC}=1$

Figure 3

TABLE 8: Experiment results comparison under luminance attacks (NC values).

\begin{tabular}{lcccc}
\hline Luminance & $\begin{array}{c}20 \% \\
\text { Brighter }\end{array}$ & $\begin{array}{c}40 \% \\
\text { Brighter }\end{array}$ & $\begin{array}{c}20 \% \\
\text { Darker }\end{array}$ & $\begin{array}{c}40 \% \\
\text { Darker }\end{array}$ \\
\hline $\begin{array}{l}\text { Zhu and Sang } \\
\text { [14] }\end{array}$ & 0.5224 & 0.6928 & 0.7484 & 0.5264 \\
$\begin{array}{l}\text { Xiao et al. [16] } \\
\text { Leung et al. [17] }\end{array}$ & 0.9926 & 0.9926 & 0.9926 & 0.9926 \\
$\begin{array}{l}\text { Tao and } \\
\text { Eskicioglu [18] }\end{array}$ & 0.9505 & 0.9505 & 0.0273 & N/A \\
$\begin{array}{l}\text { Ni et al. [19] } \\
\begin{array}{l}\text { Proposed } \\
\text { scheme }\end{array}\end{array}$ & 1 & 0.9329 & 1 & 1 \\
\hline
\end{tabular}

TABLE 9: Experiment results comparison under contrast attacks (NC values).

\begin{tabular}{lcccc}
\hline Contrast & $\begin{array}{c}20 \% \\
\text { Increase }\end{array}$ & $\begin{array}{c}40 \% \\
\text { Increase }\end{array}$ & $\begin{array}{c}20 \% \\
\text { Decrease }\end{array}$ & $\begin{array}{c}30 \% \\
\text { Decrease }\end{array}$ \\
\hline Zhu and Sang & 0.618 & 0.5143 & 0.7783 & 0.4869 \\
[14] & 0.9926 & 0.9926 & 0.9926 & 0.9926 \\
Xiao et al. [16] & 1 & 1 & 1 & 1 \\
$\begin{array}{l}\text { Leung et al. [17] } \\
\text { Tao and }\end{array}$ & 0.6041 & 0.5742 & 0.8297 & 0.6995 \\
Eskicioglu [18] & 1 & 1 & 0.976 & 0.6809 \\
Ni et al. [19] & 1 & 0.9662 & 0.9963 & 0.9888 \\
Proposed scheme & 1 & & &
\end{tabular}

watermarking schemes which are proposed by Zhu and Sang [14], Xiao et al. [16], Leung et al. [17], Tao and Eskicioglu [18], and Ni et al. [19]. Tables 1-10 show the performance of these watermarking schemes in term of the normalized crosscorrelation values and PSNR values. The attacked images are presented in Figure 4 with the parameters used for different attacks.
TABLE 10: Experiment results comparison under median filtering and histogram equalization (NC values).

\begin{tabular}{lcc}
\hline Attacks & Median filtering $(3 \times 3)$ & $\begin{array}{c}\text { Histogram } \\
\text { equalization }\end{array}$ \\
\hline Zhu and Sang [14] & 0.9889 & 0.5058 \\
Xiao et al. [16] & 0.9742 & 0.9927 \\
Leung et al. [17] & 1 & 1 \\
Tao and Eskicioglu [18] & 0.9232 & 0.8877 \\
Proposed scheme & 0.9926 & 0.9926 \\
\hline
\end{tabular}

From Table 1, we can see that the PSNR value of watermarked image using our proposed method is $40.379 \mathrm{~dB}$, which is comparable to other watermarking schemes. This indicates that the proposed watermarking scheme has good visual fidelity. Zhu's scheme obtains the best watermarked image quality, while Tao's scheme is the worst one.

For the Gaussian noises attacks, the proposed scheme outperforms Tao's and Zhu's schemes but is little worse than other schemes as shown in Table 2. From Tables 3 and 4 , it can be seen that the proposed method is not robust against the salt and pepper noises and Laplacian sharpening. Compared with Zhu's, Xiao's, Leung's, and Tao's schemes, it is observed that there is higher robustness to JPEG compression with the proposed scheme. Related results are shown in Table 5. Besides, for low-pass filtering, it is observed that the robustness of proposed method is relatively better than Zhu's, Tao's, and Xiao's algorithms when the window size and variance are small, where the NC values are closed to 1 as shown in Table 6. For cropping attacks, our proposed method generally outperforms other watermarking schemes in all cases except the Zhu one which is summarized in Table 7 . Tables 8 and 9 highlight the results achieved for luminance and contrast attacks. From the results, the proposed method outperforms other four algorithms except the Leung one, where the $\mathrm{NC}$ values are about 0.8 to 1 . Table 10 shows that the proposed method 


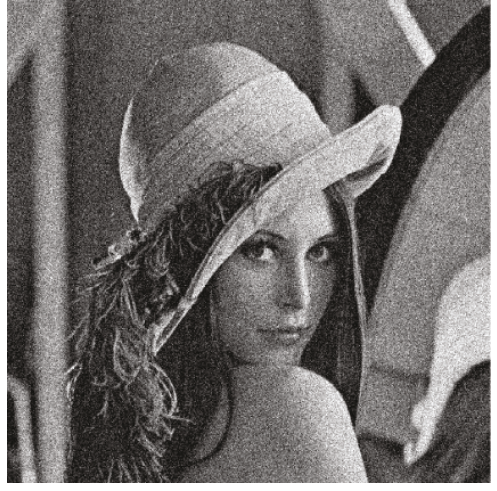

(a) Guassian noises (Standard variance $=$ 30)

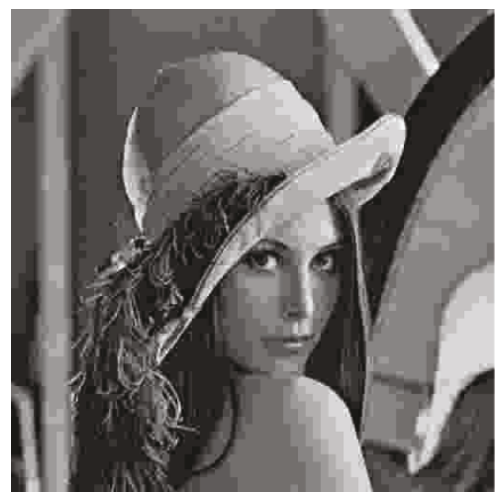

(d) Jpeg compression $(\mathrm{QF}=5)$

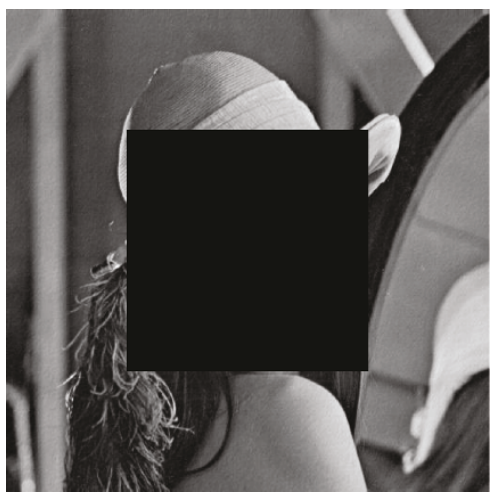

(g) Cropping (Type 2)

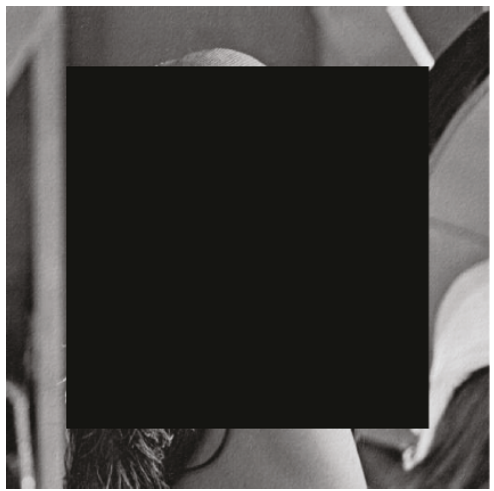

(j) Cropping (Type 5)

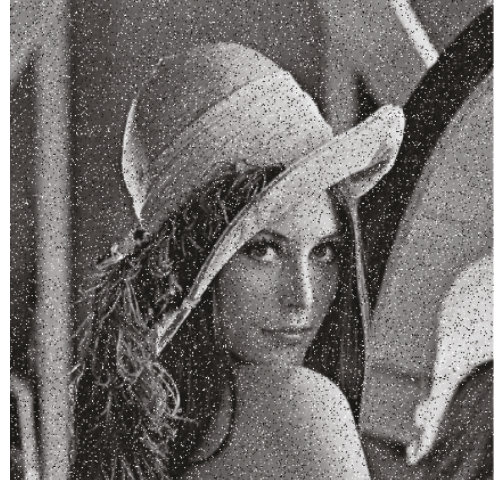

(b) Salt and pepper noises

(Density parameter $=0.1$ )

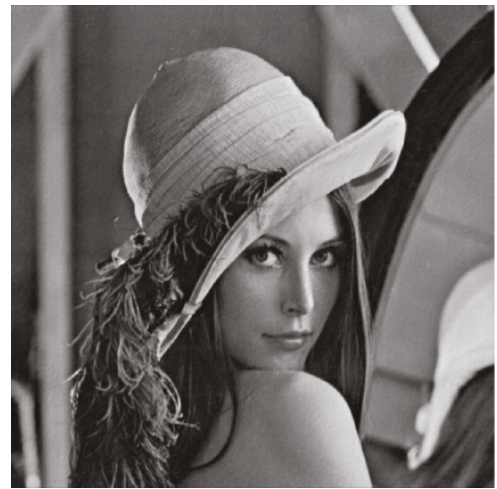

(e) Low-pass filtering (Standard variance (window) equal 0.5(5))

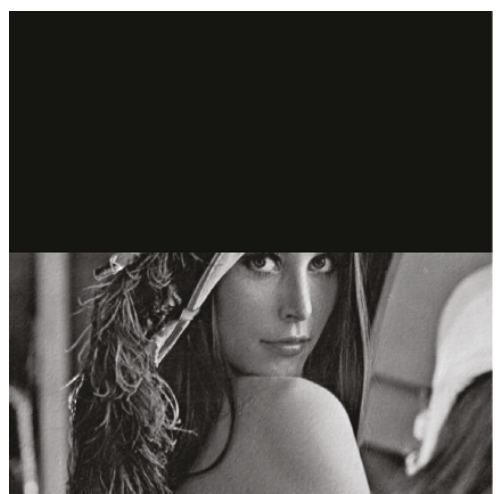

(h) Cropping (Type 3)

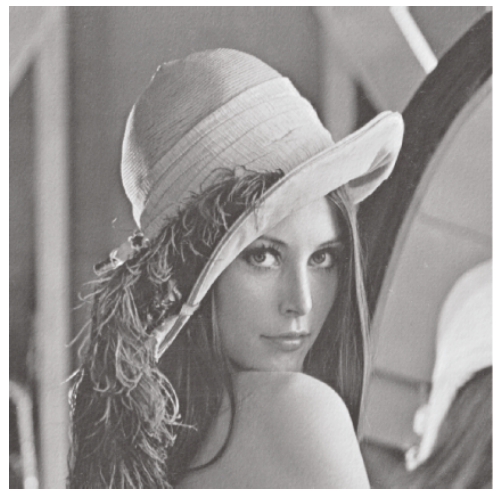

(k) $40 \%$ Brighter

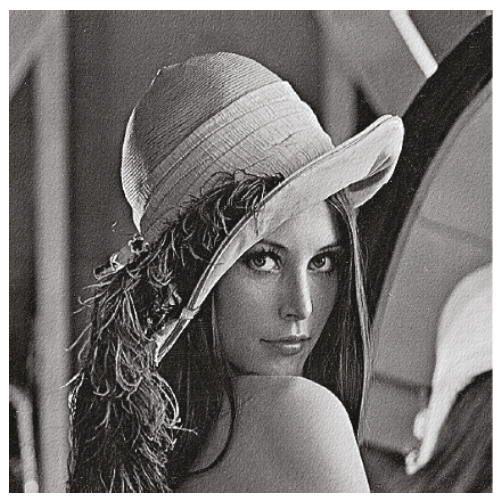

(c) Laplacian sharpening (parameter = $0.1)$

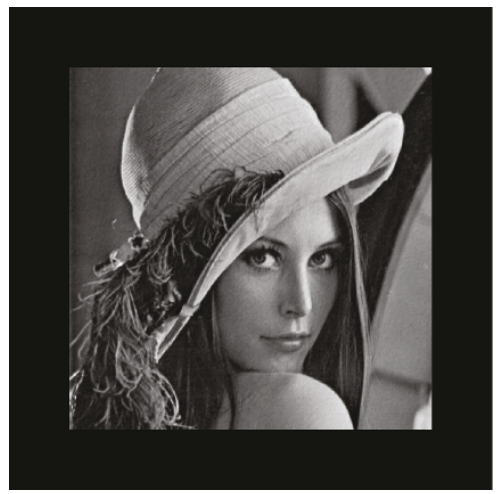

(f) Cropping (Type 1)

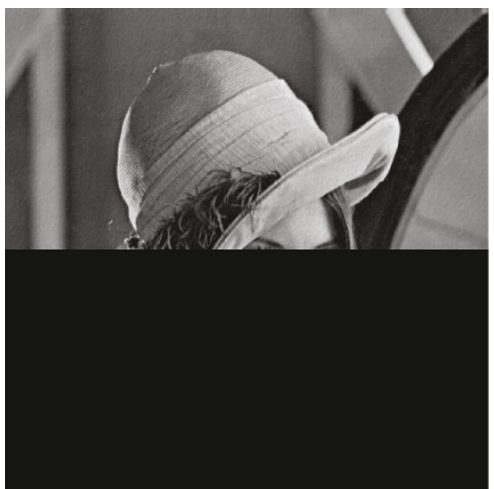

(i) Cropping (Type 4)

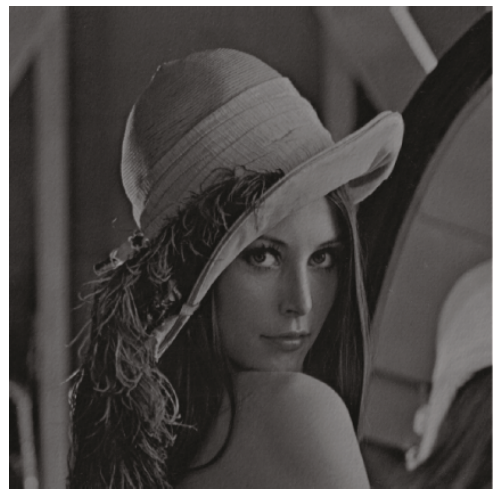

(1) $40 \%$ Darker

Figure 4: Continued. 


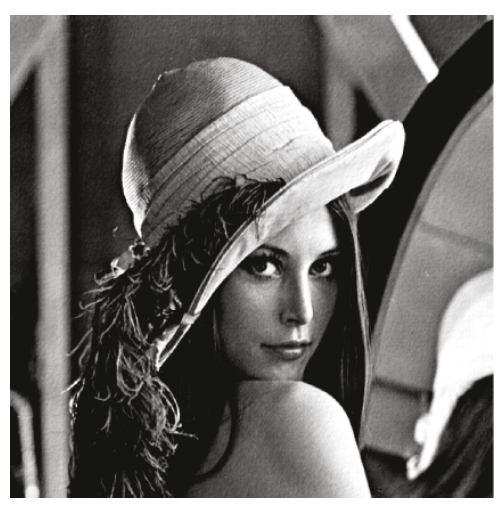

(m) $40 \%$ Contrast increase

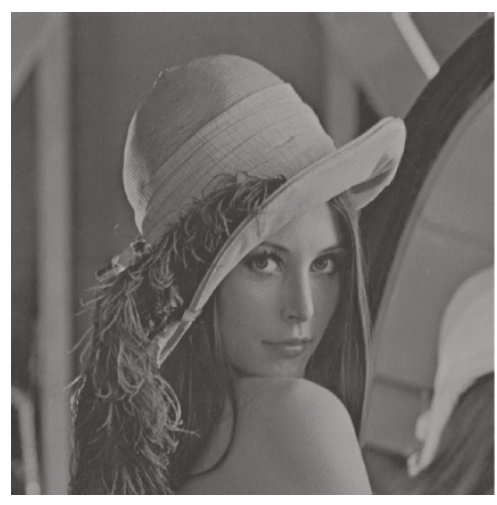

(n) $30 \%$ Contrast decrease

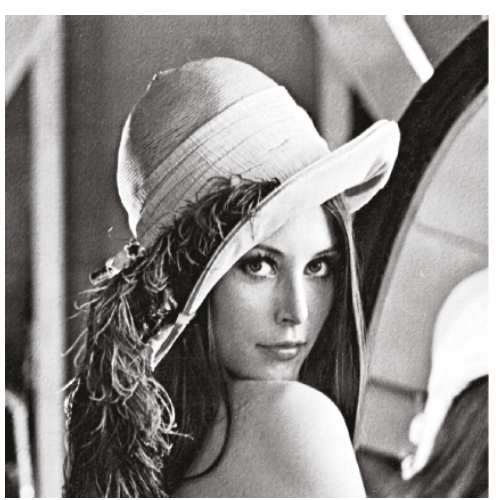

(p) Histogram equalization

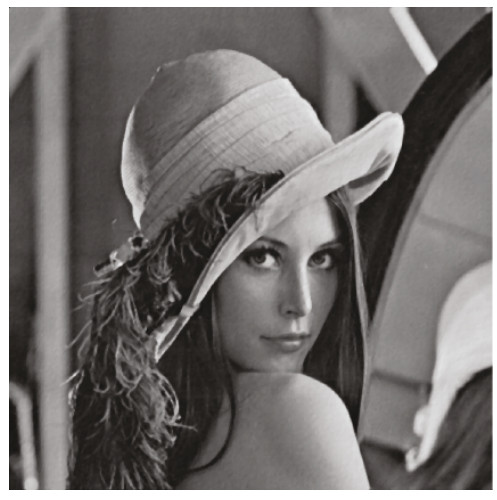

(o) Median filtering

FIgURE 4: Attacks on the watermarked image Lena.

is more robust than Zhu's, Xiao's, and Tao's algorithms for median filtering and histogram equalization.

Besides, we also performed some numerical experiments with other gray-scale standard images such as "Boat", "Pepper", and "Airplane". The PSNR values for all watermarked images are over $40 \mathrm{~dB}$. Most simulation results are the same as using the image "Lena" except histogram equalization. The watermark of the proposed method only survives histogram equalization in images "Lena" and "Pepper". For the images "Boat" and "Airplane", the NC values are only 0.6886 and 0.4503 , respectively.

Table 11 summarizes the processing time for watermark embedding and retrieval. Image Lena is used. It shows that the processing time of our proposed scheme is longer than that of Zhu's scheme but shorter than those of other four schemes, which are $2.03 \mathrm{~s}$ and $2.07 \mathrm{~s}$ for embedding and extracting, respectively. The processing time of proposed scheme is acceptable compared with other watermarking schemes. Overall, our proposed method achieved relatively better performance than those of Zhu and Sang [14], Tao and Eskicioglu [18], and $\mathrm{Ni}$ et al. [19] and obtained great robustness.

\section{Conclusion}

In this paper, a robust watermarking scheme based on the wave-atom transform is presented. The watermark is
TABLE 11: The processing time for watermark embedding and retrieval.

\begin{tabular}{lcc}
\hline & $\begin{array}{c}\text { Processing time for } \\
\text { watermark } \\
\text { embedding (s) }\end{array}$ & $\begin{array}{c}\text { Processing time for } \\
\text { watermark retrieval } \\
(\mathrm{s})\end{array}$ \\
\hline Zhu and Sang [14] & 1.02 & 0.38 \\
Xiao et al. [16] & 6.22 & 2.31 \\
Leung et al. [17] & 6.41 & 5.37 \\
Tao and Eskicioglu [18] & 0.9 & 9.45 \\
Proposed scheme & 2.23 & 2.07 \\
\hline
\end{tabular}

embedded in the wave-atom domain of four subimages. The watermark extraction process is simple and does not need the original image. The main idea of our proposed method is based on adjusting the coefficient modulus after division. The quality of the watermarked image is good in terms of perceptibility and PSNR (over $40 \mathrm{~dB}$ ). By comparing with other watermarking schemes, the experimental results show that our proposed method is more robust against attacks such as JPEG compression, median filtering, Gaussian filtering, cropping, luminance, and contrast attacks, but it fails against salt and pepper noises and sharpening attacks. The results show that the proposed method outperforms the DCT [14], wavelet [18], iterative mapping [19], and blind curvelet [16] and as expected works slightly worse 
than the curvelet nonblind approaches [17]. To conclude, from the experimental results, it is believed that digital watermarking using wave atom is able to obtain great robustness.

\section{References}

[1] C. I. Podilchuk and E. J. Delp, "Digital watermarking: algorithm and application," IEEE Signal Processing Magazine, vol. 18, no. 4, pp. 33-46, 2001.

[2] I. J. Cox, J. Kilian, F. T. Leighton, and T. Shamoon, "Secure spread spectrum watermarking for multimedia," IEEE Transactions on Image Processing, vol. 6, no. 12, pp. 1673-1687, 1997.

[3] S. Katzenbeisser and F. A. P. Petitcolas, Information Hiding Techniques for Steganography and Digital Watermarking, Artech House, Boston, Mass, USA, 2000.

[4] C.-S. Shieh, H.-C. Huang, F.-H. Wang, and J.-S. Pan, "Genetic watermarking based on transform-domain techniques," Pattern Recognition, vol. 37, no. 3, pp. 555-565, 2004.

[5] F. Y. Shih and S. Y. T. Wu, "Combinational image watermarking in the spatial and frequency domains," Pattern Recognition, vol. 36, no. 4, pp. 969-975, 2002.

[6] P. Tao and A. M. Eskicioglu, "A robust multiple watermarking scheme in the discrete wavelet transform domain," in Internet Multimedia Management Systems V, Proceedings of SPIE, pp. 133-144, Philadelphia, Pa, USA, October 2004.

[7] L. Demanet, Curvelets, wave atoms, and wave equations, Ph.D. thesis, Caltech, 2006, http://math.stanford.edu/ laurent/ papers/ThesisDemanet.pdf.

[8] L. Demanet and L. Ying, "Wave atoms and sparsity of oscillatory patterns," Applied and Computational Harmonic Analysis, vol. 23, no. 3, pp. 368-387, 2007.

[9] J. Rajeesh, R. S. Moni, and S. Palanikumar, "Noise reduction in magnetic resonance images using wave atom shrinkage," The International Journal of Image Processing, vol. 4, no. 2, pp. 131$141,2010$.

[10] A. Federico and G. H. Kaufmann, "Denoising in digital speckle pattern interferometry using wave atoms," Optics Letters, vol. 32, no. 10, pp. 1232-1234, 2007.

[11] A. S. Lewis and G. Knowles, "Image compression using the 2D wavelet transform," IEEE Transactions of Image Processing, vol. 1, no. 2, pp. 244-250, 1992.

[12] L. Demanet and L. Ying, "Wave atoms and time upscaling of wave equations," Numerische Mathematik, vol. 113, no. 1, pp. $1-71,2009$.

[13] http://www.waveatom.org/.

[14] G. Zhu and N. Sang, "Watermarking algorithm research and implementation based on DCT block," World Academy of Science, Engineering and Technology, vol. 45, pp. 38-42, 2008.

[15] B. Chen and G. W. Wornell, "Quantization index modulation: a class of provably good methods for digital watermarking and information embedding," IEEE Transactions on Information Theory, vol. 47, no. 4, pp. 1423-1443, 2001.

[16] Y. Xiao, L. M. Cheng, and L. L. Cheng, "A robust image watermarking scheme based on a novel HVS model in curvelet domain," in Proceedings of the 4th International Conference on Intelligent Information Hiding and Multimedia Signal Processing (IIH-MSP '08), pp. 343-347, Harbin, China, August 2008.

[17] H. Y. Leung, L. M. Cheng, and L. L. Cheng, "A robust watermarking scheme using selective curvelet coefficients," International Journal of Wavelets, Multiresolution and Information Processing, vol. 7, no. 2, pp. 163-181, 2009.
[18] P. Tao and A. M. Eskicioglu, "A robust multiple watermarking scheme in the Discrete Wavelet Transform domain," in Internet Multimedia Management Systems V, vol. 5601 of Proceedings of SPIE, pp. 133-144, Philadelphia, Pa, USA, October 2004.

[19] R. Ni, Q. Ruan, and H. D. Cheng, "Secure semi-blind watermarking based on iteration mapping and image features," Pattern Recognition, vol. 38, no. 3, pp. 357-368, 2005. 